\title{
Influencia de la satisfacción laboral y la percepción del abuso del poder jerárquico en el bienestar subjetivo del docente universitario
}

\section{Influence of job satisfaction and hierarchical abuse perception in university professors subjective well being}

\author{
HERNÁNDEZ-RUIZ, María Guadalupe†* \& CASTRO-VALENCIA, Alberto Merced \\ Universidad Autónoma de Guadalajara, jefatura del Departamento de Humanidades. Av. Patria 1201, Lomas del Valle, \\ 45129 Zapopan, Jal.
}

ID $1^{\text {er }}$ Autor: María Guadalupe, Hernández-Ruiz / ORC ID: 0000-0003-4725-4043

ID $1^{\text {er }}$ Coautor: Alberto Merced, Castro-Valencia / ORC ID: 0000-0001-8160-9077, Researcher ID Thomson: D-34622018, arXiv Author ID: 2776339 PubMed Autor ID: D-3462-2018, CVU CONACYT ID: 222325

\begin{abstract}
Resumen
Objetivos: Explicar la percepción del bienestar subjetivo y de la satisfacción laboral del docente ante la percepción del abuso del poder. Metodología: Se pretende realizar una correlación de variables, a través de operaciones de estadística descriptiva y ecuaciones estructurales, empleando el SPSS AMOS versión 25. Aplicar el instrumento a una población compuesta por docentes de Instituciones de Educación Superior, en la modalidad de educación privada de la Zona Metropolitana de Guadalajara con características similares de participación del mercado estudiantil, que cuenten con convenios. Otra opción mayormente viable en tiempo y recursos será en diferentes campi de la Universidad Autónoma de Guadalajara ubicados en Tabasco y Tepic. El primer instrumento se diseñó tratando de abarcar los constructos a medir, con base en la literatura revisada hasta el momento, tomando en cuenta la parsimonia y el público adecuado para el universo contemplado, asimismo, se empleó una escala 1-5 likert. Contribución: Teórica: explicar si existe un efecto moderador del BS ante la percepción de abuso y la SL en docentes universitarios, aportar a los estudios en docentes universitarios mexicanos. Metodológica: validación de instrumentos aplicados en otros contextos y sujetos. Práctica: identificar áreas de oportunidad para la implementación de programas que tiendan a favorecer la SL y reducir la percepción del abuso.
\end{abstract}

Bienestar subjetivo, Satisfacción laboral, Abuso del poder jerárquico

\begin{abstract}
Objectives: Explain the subjective well being perception and job satisfaction of the professor thru power abuse perception. Methodology: The intention is to correlate some variables, thru descriptive stadistical operations and structural equations, using SPSS AMOS 25. The questionnaire will be answered by a population of private university teachers from the metropolitan zone of Guadalajara, with similarities of market share and agreements. Another viable option in time and resources could be in different UAG campi, as in Tabasco and Tepic. The first questionnaire was designed taking in consideration the constructs to measure, according the bibliographical research until now, taking into account the parsimony and the suitable people of the study for the contemplated universe, using a 1 to 5 likert scale. Contribution: Theorical: to explain if exists a subjective well being moderator effect in light of abuse percetion and job satisfaction in university teachers; to contribute in mexican teachers papers. Methodological: validate questionnaires in different contexts and people. Practical: identify opportunity áreas for the program implementation in job satisfaction improvement and decrease abuse perception.
\end{abstract}

Subjective well being, Job satisfaction, Hierarchical power abuse

Citación: HERNÁNDEZ-RUIZ, María Guadalupe \& CASTRO-VALENCIA, Alberto Merced. Influencia de la satisfacción laboral y la percepción del abuso del poder jerárquico en el bienestar subjetivo del docente universitario. Revista de Políticas Universitarias. 2019. 3-10: 37-51.

\footnotetext{
* Correspondencia del Autor (Correo electrónico: maria.ruiz@edu.uag.mx)

$\dagger$ Investigador contribuyendo como primer autor.
} 


\section{Introducción}

Desde principios del siglo XXI, los gobiernos han mostrado interés por obtener información acerca de los estados anímicos y percepciones de los ciudadanos con relación a su bienestar y calidad de vida; de ese interés surge el informe Stiglitz- Sen- Fitoussi (2008).

Organismos internacionales como la OMS, proyectan que para el año 2020, la depresión será la causa número uno de incapacidad laboral en países en vías de desarrollo, como lo es México. Dentro de las profesiones con propensión al estrés y burnout, está la docencia. Los factores previamente mencionados, y las condiciones nacionales y mundiales urgen a investigar el estado anímico y su percepción por parte de los trabajadores. La temática del Bienestar Subjetivo ha sido investigada desde la óptica de la Psicología positiva, teniendo sus antecedentes históricos desde los escritos de Aristóteles, pasando por la Escuela Humanista de la Administración, hasta el presente siglo, donde los gobiernos instan por este tipo de estudios.

Por otro lado, el interés por la satisfacción laboral también surge en una época post industrial donde el capital humano toma preponderancia. El concepto del líder es básico para comprender a las organizaciones, siendo las escuelas y universidades un modelo sui géneris.

Con base en lo anterior, el problema se formula a partir de la cuestión: ¿De qué manera influyen la satisfacción laboral y la presencia de la justicia organizacional en el bienestar subjetivo del docente universitario?

Ya que, en el presente estudio, se plantea evaluar las variables del bienestar subjetivo satisfacción laboral y percepción del abuso del poder, a través de las siguientes dimensiones o factores: para bienestar subjetivo: satisfacción con la vida y afectos positivos y negativos; para satisfacción laboral, son seis factores; 1. Satisfacción con el cargo 2. Satisfacción con la dirección y el liderazgo 3. Satisfacción con las prestaciones recibidas 4. Satisfacción con la tarea y su ejercicio 5. Satisfacción con el ambiente y condiciones de trabajo 6 . Satisfacción con la compatibilidad del trabajo y otras actividades. Y para Percepción del abuso del poder; estilo de liderazgo, ambiente organizacional y abuso del poder.
El tipo de análisis es cuantitativo, se aplicó estadística descriptiva, pruebas estadísticas de confiabilidad, pruebas de normalidad y correlaciones entre elementos por factor. La hipótesis central, se enfoca en obtener niveles de confiabilidad superiores a 0.5 en las tres variables, de manera que:

- $\mathrm{H}_{0}$ : El nivel de confiabilidad del Alfa de Cronbach es menor a 0.5 .

- $\mathrm{H}_{\mathrm{a}}$ : El nivel de confiabilidad del Alfa de Cronbach es mayor a 0.5 .

El presente artículo se compone de seis apartados. Los cuales son:

1. Introducción, se explica de manera general el tema en estudio, así como la importancia, la técnica a utilizar, la hipótesis principal y la problemática en sí, además de las generalidades a tratar durante el artículo.

2. Marco teórico, se describen las 3 variables, bienestar subjetivo, satisfacción laboral y percepción del abuso del poder jerárquico. Se incluye también el modelo teórico propuesto, sujeto a comprobación en posteriores investigaciones.

3. Método, se incluye el tipo y diseño de investigación, la descripción conceptual y operacional de las variables tanto dependiente como independientes, además de las variables categóricas y demográficas, los instrumentos de medición, los participantes, el procedimiento y el análisis de datos.

4. Resultados y discusión, se detallan los resultados descriptivos por factor, pruebas de normalidad, correlaciones entre cada elemento y el cálculo del Alfa de Cronbach de las tres variables del estudio.

5. Anexos, se muestran los ítems del instrumento.

6. Agradecimientos, se reconoce a los participantes por su aportación.

7. Conclusiones y recomendaciones, se explican los principales hallazgos y trabajo a futuro.

8. Referencias, se muestra la bibliografía consultada. 


\section{Marco teórico}

El concepto bienestar subjetivo es un asunto prístino, el ser humano busca la felicidad y trata de conseguir aquello que lo haga feliz, el hombre ha indagado en las razones prácticas, intelectuales, morales y espirituales de la felicidad. El tema de la configuración del concepto del bienestar subjetivo se fundamenta en la Escuela de las Relaciones Humanas, la cual se interesó por el elemento humano de la empresa, Lazo Pérez, M., \& Lamanier Ramos, J (2017), junto con sus representantes, desde: Weber, E. Mayo, pasando por Maslow, Mary Parker Follet, hasta organizaciones mundiales como la ONU y la OCDE.

Dicha Escuela, integrante de las tres primigenias teorías de la Administración, en conjunto con la Teoría Científica y la Clásica, donde la primera se enfoca en la tarea y la segunda, en la estructura, sin dejar de lado, el capital humano. Pero es esta tercera, la que, con auxilio de las Ciencias Sociales, como la Psicología y la Sociología dan cuenta del valor humano en la empresa. El hombre es un ser social por naturaleza, el zoon politikon de Aristóteles libro I; es un ser diferente a los animales en la medida que crea sociedades y modifica el ambiente que habita. Martínez Crespo, 2005, en su artículo: Administración y Organizaciones. Su desarrollo evolutivo y las propuestas para el nuevo siglo, revisita las teorías de cuatro personajes, donde describe el papel del hombre en la empresa:

\begin{tabular}{|l|l|l|}
\hline Taylor & Científico & $\begin{array}{l}\text { El hombre correcto para el puesto } \\
\text { correcto }\end{array}$ \\
\hline Fayol & Clásico & Proceso Administrativo, estructuralista \\
\hline Weber & Humanista & $\begin{array}{l}\text { Actividades Sociales, éticas y de } \\
\text { dominación. La Burocracia }\end{array}$ \\
\hline Mayo & Humanista & $\begin{array}{l}\text { Hombre social, los individuos no son } \\
\text { seres aislados, sino miembros de una } \\
\text { diversidad de grupos, presencia de } \\
\text { grupos y de líderes informales. }\end{array}$ \\
\hline
\end{tabular}

Tabla 1 Comparativo de representantes de las Escuelas Administrativas

Fuente: elaboración propia (2019), con información de Martínez Crespo (2005)

Dadas las condiciones de la época, y las situaciones vividas en la mayoría de las fábricas, aparece Robert Owen como visionario y utópico, Santos Redondo, (2000) Es predecesor de las teorías de la Escuela de Relaciones Humanas, aplica sus ideas en dos empresas, New Lannark y New Harmony con resultados contrastantes.
"Sus escritos politicos y su actividad pública comenzaron después de 1812, cuando creyó haber demostrado en la práctica, en sus fábricas, que un trato más humanitario a los trabajadores era un incentivo mucho más efectivo que el castigo o incluso que el mero aumento de salario. No es difícil reconocer aquí los planteamientos de Elton Mayo y su Escuela de Relaciones Humanas frente al taylorismo, más de un siglo después; Owen es sin duda un pionero en la teoría y en la práctica del management moderno."

Santos Redondo, Manuel. "Robert Owen empresario" Revista empresa y humanismo. 2003, VOL. 6, No. 1, PÁG.179-198.

\section{Definiciones del Bienestar Subjetivo}

Carol D. Ryff and Corey Lee M. Keyes 1995, aluden a la obra de Bradburn, N. M. 1969. The Structure of Psychological Well-Being. En su estudio intitulado: The Structure of Psychological Well-Being Revisited. Rastrean el término Well- being, o bienestar subjetivo a partir de las operacionalizaciones de Bradburn, así como uno de los principales indicadores del BS, como la satisfacción con la vida.

Diener, 1984; desarrolló un modelo en el cual el BS está compuesto por influencias negativas, (NA), positivas (PA), y satisfacción con la vida; asimismo, propuso dos instrumentos para medirlos, el: SWLS, 1985 y el SPANE, 2009. Diener, Lucas y Oshi, 2002; lo definen como "la evaluación cognitiva y afectiva de la vida de una persona"

Prieto, E. Diener, Tamir, Scollon y M.Diener, 2005; presentan los componentes del constructo: eventos y circunstancias, reacciones emocionales, memoria y emociones y juicios globales, los cuales a su vez se ven afectados por: reacciones afectivas, metas y deseos, teorías de la emoción, normas culturales y estado anímico. Para ellos, el BS se relaciona con la preocupación de las personas acerca de la evaluación de sus vidas desde todos los ángulos, pasando por el instante más breve a situaciones crónicas que tienen que ver con sus estados de ánimo y los sentimientos provocados. El BS tiene total relación con el concepto de la felicidad, sumamente cualitativo, con elementos tanto materiales como intangibles, personales e interpersonales. 
Además, este grupo ha identificado las distintas vertientes o enfoques desde los que se ha estudiado el BS.

Uno de los primeros enfoques, y más general, es el que toma al BS como una evaluación global de la vida, en cuanto a la satisfacción y calidad, se puede ejemplificar con el BIARE, bienestar autor reportado, encuesta que el INEGI pone a disposición de cualquier usuario que desee responder.

Otra aproximación al BS se da desde las experiencias pasadas del individuo en un determinado lapso de su vida, lo cual se aprecia con Bradburn, 1969.

Una más se da cuando los investigadores preguntan el sentir de las personas en varios momentos de un periodo, puede ser en un día, en una semana o en lo que requiera la investigación, el resultado se obtiene mediante un promedio.

Así como los enfoques de BS son variados, los instrumentos y las medidas también lo son, los tamaños de las muestras van desde internacionales, Musikanski y Polley, 2016; hasta pequeños grupos con características demográficas específicas. Tumkaya, 2011; estudiantes universitarios, Millán, Calvanese y D' Aubeterre, 2017, profesores.

Diener et. Al, 2016; señalan algunos hallazgos para el estudio del BS, definiéndola como una nueva ciencia. La felicidad es un concepto complejo, subjetivo, al que la ciencia rehúye por atribuírsele múltiples significados, en cambio el BS, es a pesar de su nombre, un constructo identificable con la propia evaluación de la vida, en su satisfacción y calidad, siendo susceptible de medición en conjunto o individualmente hablando de otras variables.

Entre otros investigadores, como Beytía y Calvo, 2011, el concepto de "felicidad" y su estudio, es un asunto de interés general para varios sectores de la sociedad y la industria, en pleno siglo XXI, la preocupación creciente por considerar el bienestar no sólo económico, sino psicológico de las personas dentro de la organización.
Asimismo, como antecedente y elemento integrante del SWB o BS, se encuentra la rama de la Psicología Positiva, específicamente, con Martin Seligman y su discurso inaugural como presidente de la American Psychological Association (APA) (Seligman, 1999) Quien coloca en el mapa científico un concepto complejo cual es el estado humano de la felicidad, y algunos de sus componentes como: esperanza, sabiduría, creatividad, valentía, espiritualidad, perseverancia, resiliencia entre otros (PsycINFO Database 2016 APA)

Los autores en la revista American Psychologist, Vol 55(1), Enero 2000, 5-14 hacen referencia y discuten acerca de los temas o asuntos que posibilitan el sentimiento, realizan un marco de trabajo para esta ciencia, buscando llenar huecos en el conocimiento que lleven a los profesionales a comprender y a construir esos elementos que permitan al hombre y a las sociedades, crecer y desarrollarse exitosamente. Alrededor de 1995, la empresa Gallup comenzó con su proyecto $\mathrm{Q} 12$, una encuesta registrada, con una serie de doce preguntas, tales como: ¿ha recibido algún tipo de reconocimiento, ¿sabe lo que se espera de usted en el trabajo? Ha estado realizando esas encuestas desde entonces y obtenido una base de datos considerable.

Beytía y Calvo, 2011, hablan del interés por medir la felicidad, a través de diversos instrumentos propuestos por organismos internacionales cuyo propósito es orientar las políticas públicas precisamente para generar la satisfacción o bienestar subjetivo de los individuos. Dejan la puerta abierta al contenido de los instrumentos utilizados para dichas mediciones y recomiendan incluir un conjunto de tres medidas mínimas como: felicidad global, satisfacción con la vida y escalas de afectos positivos y negativos.

Las comparaciones y relaciones con otros conceptos no se hacen esperar, se ha relacionado la felicidad con el dinero, en estudios de organismos internacionales, cuando hablan de países de primer mundo y las razones del por qué han logrado ese sitio, la OCDE, (2017), tiene un "Better life index" el cual presenta en su sitio web. Examina cómo ha cambiado la vida desde el 2005, observándose que las condiciones han mejorado en algunos indicadores, pero en otros se han rezagado. 
La OCDE trabaja con once indicadores, Vivienda, Ingresos, Empleo, Comunidad, Educación, Medio Ambiente, Compromiso cívico, Salud, Satisfacción, Seguridad y Balance vida-trabajo. Otro organismo no gubernamental, nacional, dado a la tarea de medir y cuantificar, el INEGI, también ha investigado las percepciones de los mexicanos acerca de la felicidad, basándose en los indicadores señalados por la entidad internacional con el término BIARE, Bienestar autorreportado.

El INEGI fue uno de los primeros organismos de estadística en el mundo en implementar este tipo de mediciones. Con una primera aplicación exploratoria, a la cual denominaron BIARE Piloto, realizada en 2012, en conjunto con la Encuesta Nacional de Gastos de los Hogares ENGASTO.

Dos años después, se volvió a aplicar el instrumento BIARE, que ahora se denominó Ampliado, con una muestra mayor y combinado con el Módulo de Condiciones Socioeconómicas 2014, para asociar el bienestar subjetivo con indicadores del bienestar objetivo. INEGI, boletín de prensa (2016).

Krueger y Schkade (2007), se preguntan sobre la confiabilidad de las dobles mediciones o doble aplicación de las pruebas de los índices de la felicidad a través del método del diario, experimentando con un mismo grupo de personas, respondiendo al mismo examen en dos momentos distintos en el tiempo. Por lo tanto, se percibe un interés genuino en estudiar los mecanismos del ser humano sano en sus múltiples dimensiones y necesidades.

González Villalobos y Marrero, 2015 y 2017 estudian a la población mexicana tanto en el bienestar subjetivo y psicológico, y hacen un estudio transcultural con España, así que hacen la división entre dos grandes dimensiones, lo subjetivo y lo psicológico, midiéndolos a través de varios instrumentos, algunos de los cuales se presentan a continuación:

SWLS- Escala de Satisfacción con la vida- Diener, Emmons, Larson y Griffin. 1985 SPANE- Escala de experiencias positivas y negativas- Diener, E., Wirtz, D., Tov, W., KimPrieto, C., Choi. D., Oishi, S., y Biswas-Diener, R. 2009PANAS- Escala de afectos positivos y negativos- Watson, Clark y Tellegen, 1988.
ACSA- Anamnestic comparative selfassessment- Autoevaluación comparativa de la anamnesis.

Escala de bienestar psicológico Ryff,1989, WHOQOL-100 Y WHOQOL-BREF Instrumento de calidad de vida de la Organización Mundial de la Salud, OMS, World Health Organization Quality of Life Questionnaire; WHOQOL Group, 1993.

Una variedad de investigadores ha volcado sus esfuerzos en la población docente, para conocer sus índices de BS, además de estudiar las correlaciones con un sinnúmero de constructos, dimensiones o variables, Censkeven- Onder, 2009; lo relaciona con la calidad de vida académica y el síndrome de burnout, Berrocoso, Fernández y Revuelta, 2013, nos hablan de docentes que se consideran innovadores al implementar las TICs en su quehacer diario, lo que les reporta un mayor índice de BS, reflejado en la manifestación de su satisfacción laboral.

Por otro lado, Muñoz, Fernández, 2016, señala que el entorno de la enseñanza ha sido considerado uno de los ámbitos laborales donde los trabajadores tienen mayor involucramiento y ejercicio emocional; verifica la relación entre las variables inteligencia emocional, satisfacción laboral y el BS; todas estas en nivel auto percepción, encontrando que los índices de inteligencia emocional son elementos que favorecen al BS.

Herrera y Perandones, 2017, relacionan el BS del docente con su personalidad y sentido del humor, concluyendo que son factores para tomar en cuenta en su desarrollo profesional.

Entre otros elementos de interés, se encuentra el estudio de los ambientes laborales del docente, tanto el público, como el privado, las condiciones de trabajo y las fuentes de estrés, comprobándose que el docente percibe condiciones más adversas dentro de una dependencia de gobierno, Millán y Calvanese, 2017, pero se atenúan debido al mantenimiento de una buena relación profesor- alumno, localizándose algunos factores de riesgo que inciden en el BS del docente. 


\section{Condiciones laborales y BS}

La división del trabajo apareció desde la formación de los grupos humanos, al cohabitar personas con habilidades y destrezas diferentes que permitieron el desarrollo de beneficios y comodidades. A la par de la evolución de las sociedades, surgen oficios cada vez más complejos para satisfacer las crecientes necesidades. Igualmente, se manifiesta el poder en tales relaciones, como formas primitivas de gobierno, desde la cooperativa hasta la esclavitud.

Viéndose al trabajador unas veces como mero instrumento, otras con total dignidad, en todas las edades históricas. Siendo ya evidente el interés por mejorar las condiciones del trabajador a partir de la Revolución industrial del s. XVIII, la creación de organismos internacionales como la Organización de las Naciones Unidas (ONU), la Organización Internacional del Trabajo (OIT), y la formulación de leyes que expresamente protegen al trabajador, como la Ley de seguridad social, la Declaración de los Derechos Humanos, reducción de jornada laboral y regulación laboral de los menores, entre otras.

El BS, como se mencionó anteriormente, es un constructo complejo al que se le relaciona con una multiplicidad de indicadores o que predicen el mismo BS, tales como el apoyo del grupo social y significado de la vida, que, a decir de Su, Tay y Diener, 2014, no son considerados tal cual componentes del BS.

Asimismo, la satisfacción con la vida parece recibir influencia directa de factores constantes o coincidentes en la vida cotidiana, tales como la salud, el ingreso económico y la calidad de vida laboral.

La Psicología organizacional toma en cuenta la multielementariedad del BS, así como su adaptabilidad a los distintos escenarios, tal que, dentro de una organización, la satisfacción con la vida es fácilmente equiparable a la satisfacción con el trabajo y minuciosamente desglosado en componentes específicos como el estrés laboral, además, las actitudes del trabajador son indicadores que facilitan la evaluación de la calidad de las experiencias y el ambiente profesional.
Pollicino,1996, define la satisfacción laboral del docente universitario como el grado en que perciben la acción de la Institución para proveer un clima que asegure la autonomía académica y la congruencia de las responsabilidades de acuerdo con su perfil profesional.

Jones, 2006, encuentra que la satisfacción con la vida tiene mayor influjo sobre el desempeño del empleado que con la satisfacción laboral. En retrospectiva, Vroom lo "descubrió" en 1964, a partir de la revisión de veinte estudios, una correlación positiva entre estas variables, no tan fuerte como se creía. Otros estudios posteriores confirman lo encontrado por Vroom; en cambio, Wright y Cropanzano, 2000, comentan que son necesarias más investigaciones y, sobre todo, hacer un especial énfasis al momento de operacionalizar las variables de satisfacción con la vida.

Laca, Mejía y Gondra, 2006, proponen un modelo para evaluar el bienestar laboral (BL) como elemento de la salud mental, basados en Warr 1990, 2003. Realizando una crítica a los abordajes de la Psicología en lo que a estados mentales saludables se refiere. Asimismo, se basan en diversos autores para establecer las variables e indicadores del BL.

Padilla, Jiménez y Ramírez, 2008, consideraron medir la satisfacción del docente en dos vertientes, global y por facetas, de acuerdo con Galaz, 2003. Asimismo, utilizaron el modelo de Hagedorn, 2000, que consiste en la hipótesis de la existencia de dos tipos de constructos que interactúan e influyen en los "desencadenantes", (llamados por ella triggers), y moderadores del BL, definiendo los desencadenantes como eventos significativos de la vida, pudiendo o no estar directamente relacionados con la vida laboral.

El otro constructo mediador o moderador es la variable que interactúa en las relaciones de otras variables, como cambios y una vastedad de situaciones concernientes al contexto que le dan significado al BL. Hagedorn, 2000, considera seis desencadenantes. 


\section{Liderazgo y BS}

La interacción de dos o más personas, o de una persona y un grupo de personas, o entre grupos, se ve afectada por los roles que adoptan los individuos y sus diferentes perfiles. Desde la historia de la humanidad, se ha dado generalmente de forma natural el papel a jugar en el grupo social. Así como en los inicios del gobierno, reinaba la ley del más fuerte, el papel del líder va evolucionando de la mano del poder. Uhl-Bien, Marion y Mc Kelvey, 2007, hacen una revisión del papel del líder y del liderazgo en el periodo comprendido entre la Revolución industrial y la denominada era del conocimiento. Propiamente establecen que los modelos de liderazgo del siglo pasado se enfocaban en una preferencia económica o de producción, en cambio, actualmente se requiere modificar esos esquemas por otros que estén orientados a la economía de intangibles, como el aprendizaje, la innovación y la adaptación al cambio.

\section{Modelo teórico}

El modelo corresponde al establecimiento de relaciones entre los constructos: Bienestar subjetivo (BS), Satisfacción laboral (SL) y Percepción del abuso del poder jerárquico (AP) entre una población de docentes de Instituciones de Educación Superior.

En esta propuesta, el bienestar subjetivo es la variable Dependiente, se relaciona directamente con los otros dos constructos, percepción del abuso del poder y satisfacción laboral; sujeta a comprobación en estudios posteriores, sólo se propone como sustento teórico.

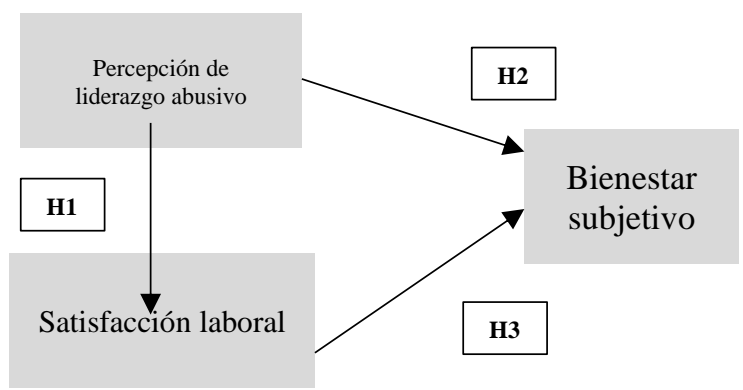

Figura 1 Modelo teórico particular, sujeto a comprobación

Fuente: elaboración propia (2019)

\section{Método}

\section{Tipo y diseño de investigación}

El esudio es de corte cuantitativo no experimental con enfoque transversal correlacional (Hernández, Fernández y Baptista, 2014; y Bernal, 2016).

\section{Variables}

\section{Variable dependiente: Bienestar Subjetivo (BS)}

Definición conceptual: es la valoración que las personas hacen de su vida y de su estado anímico. Es la evaluación cognitiva y afectiva de la vida de una persona. Diener, (1985)

Definición operacional: Está compuesto por la medición de la Satisfacción con la vida, Afectos positivos y Afectos negativos. Los cuales a su vez implican eventos y circunstancias, reacciones emocionales, memoria, emociones, juicios globales.

Afectados por: reacciones afectivas, metas y deseos, teorías de la emoción, normas culturales y estado anímico. Ed Diener, (1984) Diener, Lucas, \& Oshi, (2002).

\section{Variable independiente: Satisfacción Laboral (SL)}

Definición conceptual: Locke, (1976) la define como un estado emocional positivo o placentero resultante de una percepción subjetiva de las experiencias laborales del individuo.

Shermerhom, Hunt y Osborn, (1987) la definen como el grado en que un individuo experimenta sentimientos positivos o negativos por su trabajo; es una respuesta emocional a las tareas desempeñadas, así como a las condiciones físicas y sociales del lugar de trabajo.

Así, puede decirse que la satisfacción laboral representa una actitud del individuo hacia su trabajo, que no es otra cosa que un juicio evaluativo que refleja su experiencia pasada y presente, incluyendo su experiencia con el ambiente físico. 
Definición operacional: Pujol, Cols, (2016) mide la satisfacción laboral del docente universitario a través de un instrumento ad hoc donde a través de 28 preguntas extrae la dimensión cognitiva y el componente afectivo.

\section{Variable independiente: Percepción de abuso de poder jerárquico (PA)}

Definición conceptual: Los líderes abusivos desarrollan constantemente comportamientos agresivos como culpar a los seguidores por lo que no hicieron, ser grosero y mostrarse molesto con los seguidores por lo que alguien más le hizo (Tepper, 2007). Los estudios han demostrado que el liderazgo abusivo tiene efectos perjudiciales sobre los seguidores, su satisfacción y niveles elevados de frustración (Ashforth, 1997; Keashly, Trott y MacLean 1994). Las investigaciones han demostrado que el resultado de la agresión interpersonal en este liderazgo es el burnout. Definición operacional: Vrenderburgh y Brender 1998. Falta de respeto a la dignidad de un subordinado.

\section{1.- Percepción hacia el líder.}

2.- Imposición de cooperaciones ilegales.

3.- Intimidación.

4.- Hostigamiento físico o verbal.

5.- Avergonzar en público.

6.- Rumores destructivos.

7.- Manipulación de dependencia.

8.- Promesas falsas.

\section{Variables categóricas}

Comprenden datos demográficos, cuyas medidas corresponden a una escala de medición individualizada para cada una: sexo, edad, estado civil. Así como datos laborales como: área de desempeño, antigüedad en la Institución.

\section{Instrumentos de medición}

El instrumento piloto es resultado de la unión de 4 cuestionarios íntegros que corresponden a las variables estudiadas.
Para Bienestar Subjetivo (BS) se utilizó la SWLS, Escala de satisfacción con la vida y el SPANE, Escala de afectos positivos y negativos, de Diener $(1985,2008)$ Para Percepción de abuso del poder jerárquico (PA) se utilizó el instrumento de Flores, Madero y Gómez (2006). Para Satisfacción laboral (SL), se utilizó el instrumento de Pujol, Cols, (2016)

\section{Participantes (características de la muestra)}

Los docentes de tiempo completo se seleccionaron mediante el tipo de muestreo no probabilístico por conveniencia (Hernández, Fernández y Baptista, 2014; y Bernal, 2016). Enseguida se detallan las características de la muestra seleccionada (ver Tabla 2).

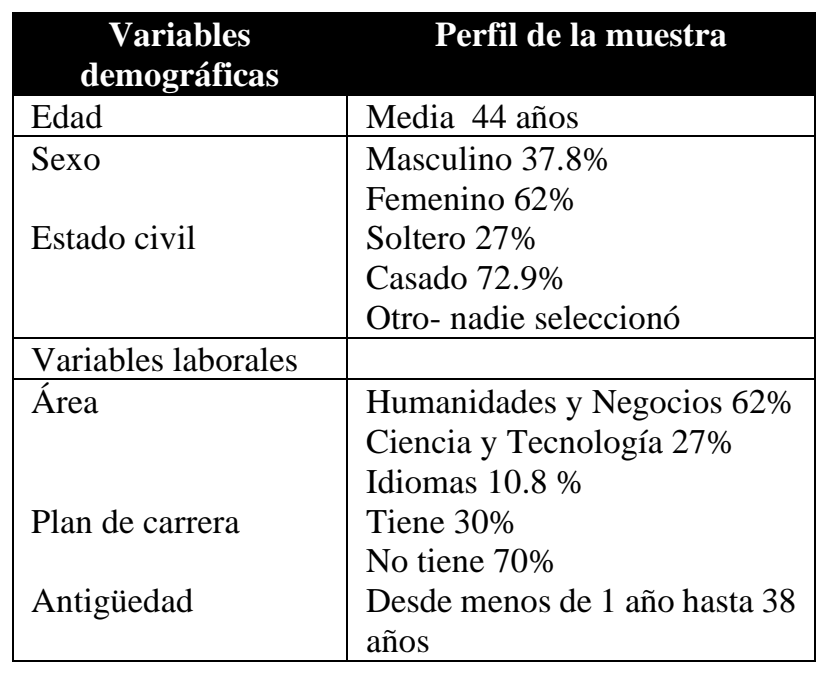

Tabla 2 Características de la muestra Fuente: elaboración propia (2019).

\section{Procedimiento}

Los datos se obtuvieron a partir de la entrega personal del instrumento impreso a tres de las áreas académicas de la Institución, fue de forma voluntaria y se recogieron en un lapso de cinco días después de entregados. El instrumento consta de 67 reactivos -sin contar las categóricas- en escala de Likert del 1 al 5, combinando conceptos de frecuencia, satisfacción y acuerdo, donde 1 indica menor frecuencia, menor satisfacción y menor acuerdo.

\section{Análisis de datos}

Se efectuaron pruebas estadísticas de confiabilidad y validez del instrumento, así como estadística descriptiva, pruebas de normalidad y correlaciones entre elementos. Los datos se procesaron con el software estadístico IBM SPSS Stadistics $®$ (versión 25) 


\section{Resultados y discusión}

\section{Resultados descriptivos}

En cuanto a los valores medios de las variables contempladas en el estudio, la mayoría fluctúa entre el 3 y el 4 . Efectos negativos NE y Liderazgo $\mathrm{L}$ se mantienen entre 2 y 3 , mientras que Percepción de abuso del poder se encuentra entre 1 y 3 . Todos con valores mínimos de 1 y valores máximos de 5

\begin{tabular}{|r|r|r|}
\hline \multicolumn{2}{|c}{ Media } & \multicolumn{2}{c|}{ Desviación } \\
\hline swl1 & 3,95 &, 524 \\
\hline swl2 & 3,59 &, 725 \\
\hline swl3 & 4,24 &, 683 \\
\hline swl4 & 4,43 &, 647 \\
\hline swl5 & 3,70 & 1,024 \\
\hline
\end{tabular}

Tabla 3 Estadística descriptiva de Satisfacción con la vida, componente del Bienestar subjetivo

Fuente: elaboración propia (2019)

\begin{tabular}{|l|r|r|}
\hline \multicolumn{2}{|c|}{ Media } & \multicolumn{2}{c|}{ Desviación } \\
\hline PA1 & 4,46 &, 605 \\
\hline PA2 & 4,24 &, 895 \\
\hline PA3 & 4,22 &, 787 \\
\hline PA4 & 4,30 &, 740 \\
\hline PA5 & 4,22 &, 854 \\
\hline PA6 & 4,24 &, 641 \\
\hline
\end{tabular}

Tabla 4 Estadística descriptiva de Afectos Positivos, componente del Bienestar subjetivo, dentro del SPANE Fuente: elaboración propia (2019)

\begin{tabular}{|r|r|r|}
\hline \multicolumn{2}{|c|}{ Media } & \multicolumn{2}{c|}{ Desviación } \\
\hline NE1 & 2,32 &, 852 \\
\hline NE2 & 2,22 &, 886 \\
\hline NE3 & 2,19 &, 938 \\
\hline NE4 & 2,22 & 1,058 \\
\hline NE5 & 2,00 & 1,130 \\
\hline NE6 & 2,65 & 1,086 \\
\hline
\end{tabular}

Tabla 5 Estadística descriptiva de Afectos Negativos, componente del Bienestar subjetivo, dentro del SPANE Fuente: Elaboración propia (2019)

\begin{tabular}{|l|r|r|}
\hline \multicolumn{2}{|c|}{ Media } & \multicolumn{1}{c|}{ Desviación } \\
\hline SL1 & 4,14 &, 822 \\
\hline SL2 & 3,68 &, 915 \\
\hline SL3 & 3,86 &, 918 \\
\hline SL4 & 3,95 &, 880 \\
\hline SL5 & 3,70 &, 812 \\
\hline SL6 & 3,95 &, 743 \\
\hline SL7 & 3,65 &, 919 \\
\hline SL8 & 3,57 & 1,068 \\
\hline SL9 & 3,73 &, 804 \\
\hline SL10 & 3,92 &, 759 \\
\hline SL11 & 3,62 &, 893 \\
\hline SL12 & 3,70 &, 996 \\
\hline SL13 & 3,97 &, 763 \\
\hline SL14 & 3,57 & 1,042 \\
\hline SL15 & 3,57 & 1,094 \\
\hline SL16 & 3,30 & 1,051 \\
\hline SL17 & 3,41 &, 927 \\
\hline SL18 & 4,19 &, 660 \\
\hline SL19 & 4,16 &, 727 \\
\hline SL20 & 4,11 &, 737 \\
\hline SL21 & 3,84 &, 764 \\
\hline SL22 & 3,30 & 1,077 \\
\hline SL23 & 3,81 & 1,101 \\
\hline SL24 & 3,59 &, 896 \\
\hline SL25 & 4,16 &, 688 \\
\hline SL26 & 4,49 &, 607 \\
\hline SL27 & 4,22 &, 787 \\
\hline SL28 & 4,27 &, 693 \\
\hline & & \\
\hline
\end{tabular}

Tabla 6 Estadística descriptiva de Satisfacción laboral Fuente: elaboración propia (2019)

\begin{tabular}{|r|r|r|}
\hline \multicolumn{2}{|c}{ Media } & \multicolumn{2}{c|}{ Desviación } \\
\hline L1 & 3,78 &, 886 \\
\hline L2 & 3,78 &, 787 \\
\hline L3 & 2,38 &, 982 \\
\hline L4 & 2,73 & 1,018 \\
\hline L5 & 2,49 &, 932 \\
\hline L6 & 2,14 &, 918 \\
\hline
\end{tabular}

Tabla 7 Estadística descriptiva de Liderazgo, componente de la Percepción de abuso del poder

Fuente: elaboración propia (2019)

\begin{tabular}{|l|r|r|}
\hline \multicolumn{3}{|c|}{ Media } \\
\hline AP1 & 1,97 &, 897 \\
\hline APx2 & 1,00 &, 000 \\
\hline APx3 & 1,08 &, 277 \\
\hline APx4 & 1,11 &, 393 \\
\hline APx5 & 1,16 &, 442 \\
\hline APx6 & 1,00 &, 000 \\
\hline APx7 & 1,14 &, 419 \\
\hline AP8 & 3,78 &, 976 \\
\hline AP9 & 3,51 & 1,070 \\
\hline AP10 & 3,84 &, 898 \\
\hline AP11 & 2,46 & 1,070 \\
\hline AP12 & 2,27 & 1,122 \\
\hline AP13 & 2,54 & 1,192 \\
\hline AP14 & 2,11 & 1,100 \\
\hline AP15 & 1,78 &, 976 \\
\hline
\end{tabular}

Tabla 8 Estadística descriptiva de Percepción de abuso del poder

Fuente: elaboración propia (2019) 


\section{Pruebas de normalidad}

Para la comprobación del comportamiento de los datos y la presencia o ausencia dentro de la curva de la normalidad, se aplicaron las pruebas cuantitativas de Asimetría y Curtosis, con estos cálculos se encontró que no todos los valores de las variables, sobre todo la variable PA, percepción de abuso; están dentro de los parámetros de normalidad, es decir, al calcular la curtosis y la asimetría se obtuvieron valores diferentes a $-1 \mathrm{y}+1$

\begin{tabular}{|c|c|c|c|c|}
\hline & \multirow{2}{*}{$\begin{array}{l}\text { Asimetría } \\
\text { Estadístico }\end{array}$} & \multicolumn{3}{|c|}{ Curtosis } \\
\hline & & $\begin{array}{l}\text { Desv. } \\
\text { Error }\end{array}$ & Estadístico & $\begin{array}{l}\text { Desv. } \\
\text { Error }\end{array}$ \\
\hline swl1 & $-1,303$ & ,388 & 5,620 &, 759 \\
\hline swl2 &,- 580 & ,388 &, 196 & ,759 \\
\hline swl3 &,- 902 & ,388 & 1,916 & ,759 \\
\hline swl4 &,- 710 &, 388 &,- 438 &, 759 \\
\hline swl5 &,- 503 & ,388 &,- 075 &, 759 \\
\hline
\end{tabular}

Tabla 9 Medidas de asimetría y curtosis de Satisfacción con la vida, de la variable Bienestar subjetivo

Fuente: elaboración propia, (2019)

\begin{tabular}{|r|r|r|r|r|}
\hline \multicolumn{2}{|c}{ Asimetría } & \multicolumn{2}{c|}{ Curtosis } \\
& Estadístico & $\begin{array}{c}\text { Desv. } \\
\text { Error }\end{array}$ & \multicolumn{1}{c|}{ Estadístico } & $\begin{array}{r}\text { Desv. } \\
\text { Error }\end{array}$ \\
\hline PA1 &,- 631 &, 388 &,- 478 &, 759 \\
\hline PA2 & $-1,745$ &, 388 & 4,217 &, 759 \\
\hline PA3 &,- 774 &, 388 &, 228 &, 759 \\
\hline PA4 &,- 982 &, 388 & 1,146 &, 759 \\
\hline PA5 & $-1,574$ &, 388 & 4,218 &, 759 \\
\hline PA6 &,- 260 &, 388 &,- 574 &, 759 \\
\hline
\end{tabular}

Tabla 10 Medidas de asimetría y curtosis de Afectos Positivos, de la variable Bienestar subjetivo

Fuente: elaboración propia, (2019)

\begin{tabular}{|r|r|r|r|r|}
\hline \multicolumn{3}{|c}{ Asimetría } & \multicolumn{2}{c|}{ Curtosis } \\
\multicolumn{2}{|c|}{ Estadístico } & $\begin{array}{c}\text { Desv. } \\
\text { Error }\end{array}$ & Estadístico & $\begin{array}{r}\text { Desv. } \\
\text { Error }\end{array}$ \\
\hline NE1 &,- 127 &, 388 &,- 736 &, 759 \\
\hline NE2 &, 054 &, 388 &,- 879 &, 759 \\
\hline NE3 &, 241 &, 388 &,- 850 &, 759 \\
\hline NE4 &, 583 &, 388 &,- 165 &, 759 \\
\hline NE5 &, 976 &, 388 &, 070 &, 759 \\
\hline NE6 &, 076 &, 388 &,- 251 &, 759 \\
\hline
\end{tabular}

Tabla 11 Medidas de asimetría y curtosis de Afectos Negativos, de la variable Bienestar subjetivo Fuente: elaboración propia, (2019)

\begin{tabular}{|c|c|c|c|c|}
\hline & \multicolumn{2}{|c|}{ Asimetría } & \multicolumn{2}{|c|}{ Curtosis } \\
\hline & Estadístico & $\begin{array}{l}\text { Desv. } \\
\text { Error }\end{array}$ & Estadístico & $\begin{array}{l}\text { Desv. } \\
\text { Error }\end{array}$ \\
\hline SL1 & $-1,214$ & ,388 & 1,817 & ,759 \\
\hline SL2 & ,021 &, 388 &,- 860 & ,759 \\
\hline SL3 &,- 403 &, 388 &,- 588 & ,759 \\
\hline SL4 &,- 666 &, 388 &, 022 & ,759 \\
\hline SL5 &,- 047 & ,388 &,- 465 & ,759 \\
\hline SL6 &,- 341 &, 388 &, 024 &, 759 \\
\hline SL7 &,- 579 & ,388 & ,699 & ,759 \\
\hline SL8 &,- 619 & ,388 &, 164 & ,759 \\
\hline SL9 &,- 134 & ,388 &,- 366 & ,759 \\
\hline SL10 &,- 666 &, 388 &, 776 & ,759 \\
\hline SL11 &,- 141 &, 388 &,- 613 & ,759 \\
\hline SL12 &,- 955 &, 388 &, 515 & ,759 \\
\hline SL13 &,- 746 &, 388 & ,957 & ,759 \\
\hline SL14 &, 044 &, 388 & $-1,157$ &, 759 \\
\hline SL15 &,- 249 &, 388 &,- 674 & ,759 \\
\hline SL16 &, 116 &, 388 & $-1,214$ & ,759 \\
\hline SL17 &, 182 &, 388 &,- 708 & ,759 \\
\hline SL18 &,- 219 & ,388 &,- 634 & ,759 \\
\hline SL19 &,- 261 & ,388 & $-1,013$ & ,759 \\
\hline SL20 &,- 615 &, 388 &, 538 &, 759 \\
\hline SL21 &,- 106 &, 388 &,- 419 & ,759 \\
\hline SL22 &,- 357 & ,388 & $-1,102$ & ,759 \\
\hline SL23 &,- 922 & ,388 & ,564 & ,759 \\
\hline SL24 &,- 545 &, 388 & ,792 & ,759 \\
\hline SL25 &,- 762 &, 388 & 1,540 & ,759 \\
\hline SL26 &,- 735 & ,388 &,- 358 & ,759 \\
\hline SL27 &,- 774 & ,388 & ,228 & ,759 \\
\hline SL28 &,- 419 &, 388 &,- 799 & ,759 \\
\hline
\end{tabular}

Tabla 12 Medidas de asimetría y curtosis de Satisfacción Laboral

Fuente: laboración propia, (2019)

\begin{tabular}{|r|r|r|r|r|}
\hline \multicolumn{4}{c}{ Asimetría } & \multicolumn{2}{c|}{ Curtosis } \\
\multicolumn{2}{|c|}{ Estadístico } & $\begin{array}{c}\text { Desv. } \\
\text { Error }\end{array}$ & Estadístico & $\begin{array}{r}\text { Desv. } \\
\text { Error }\end{array}$ \\
\hline L1 &,- 561 &, 388 &,- 154 &, 759 \\
\hline L2 &,- 313 &, 388 &,- 078 &, 759 \\
\hline L3 &, 639 &, 388 &, 203 &, 759 \\
\hline L4 &, 083 &, 388 &,- 617 &, 759 \\
\hline L5 & 1,022 &, 388 & 1,386 &, 759 \\
\hline L6 & 1,087 &, 388 & 1,737 &, 759 \\
\hline
\end{tabular}

Tabla 13 Medidas de asimetría y curtosis de Liderazgo, de la variable Percepción del abuso del poder

Fuente: elaboración propia, (2019)

\begin{tabular}{|l|r|r|r|r|}
\hline \multicolumn{2}{c}{} & \multicolumn{2}{c|}{ Asimetría } & \multicolumn{2}{c|}{ Curtosis } \\
\cline { 2 - 5 } & Estadístico & $\begin{array}{c}\text { Desv. } \\
\text { Error }\end{array}$ & Estadístico & $\begin{array}{r}\text { Desv. } \\
\text { Error }\end{array}$ \\
\hline AP1 &, 543 &, 388 &,- 515 &, 759 \\
\hline APx2 &. &. & &. \\
\hline APx3 & 3,201 &, 388 & 8,713 &, 759 \\
\hline APx4 & 3,934 &, 388 & 16,055 &, 759 \\
\hline APx5 & 2,868 &, 388 & 8,277 &, 759 \\
\hline APx6 & & & & \\
\hline APx7 & 3,328 &, 388 & 11,391 &, 759 \\
\hline AP8 &,- 676 &, 388 &, 492 &, 759 \\
\hline AP9 &,- 612 &, 388 &, 025 &, 759 \\
\hline AP10 &,- 151 &, 388 &,- 896 &, 759 \\
\hline AP11 &,- 177 &, 388 & $-1,244$ &, 759 \\
\hline AP12 &, 303 &, 388 & $-1,272$ &, 759 \\
\hline AP13 &, 158 &, 388 & $-1,168$ &, 759 \\
\hline AP14 &, 702 &, 388 &,- 238 &, 759 \\
\hline AP15 &, 841 &, 388 &,- 612 &, 759 \\
\hline & & & & \\
\hline
\end{tabular}

Tabla 14 Medidas de asimetría y curtosis de Percepción de abuso del poder

Fuente: elaboración propia, (2019) 


\section{Correlaciones}

Para propósitos de alcance de la presente investigación, en las matrices de correlaciones entre elementos se pueden observar cómo se correlacionan éstos entre sí, aunque no todos alcanzan valores mayores a 0.5 (consultar tablas de la 15 a la 20). Los resultados obtenidos muestran correlaciones débiles en la dimensión Bienestar Subjetivo BS en el componente Satisfacción con la vida, tabla15; en Afectos Positivos y negativos los valores se incrementan, tablas 16 y 17 , para decaer de nuevo en Liderazgo, componente de Abuso del poder, tabla 18, apareciendo valores negativos. En la dimensión Abuso del poder, se aprecia claramente la problemática, tabla 19 al presentarse en su mayoría, valores negativos y menores a 0.5 . En cuanto a Satisfacción laboral, tabla 20 , se aprecian valores mayores a 0.5 en su generalidad.

\begin{tabular}{|c|c|c|c|c|c|}
\hline & swl1 & swl2 & swl3 & swl4 & swl5 \\
\hline swl1 & 1,000 & & & & \\
\hline swl2 & 306 & 1,000 & & & \\
\hline swl3 & ,658 & ,205 & 1,000 & & \\
\hline swl4 & ,644 & ,266 &, 635 & 1,000 & \\
\hline swl5 & ,435 &,- 204 & ,424 & ,409 & 1,000 \\
\hline
\end{tabular}

Tabla 15 Correlación de ítems Satisfacción con la vida, componente de Bienestar Subjetivo

Fuente: elaboración propia, (2019)

\begin{tabular}{|l|r|r|r|r|r|r|}
\hline \multicolumn{2}{|c}{ PA1 } & \multicolumn{1}{c|}{ PA2 } & \multicolumn{1}{c|}{ PA3 } & \multicolumn{1}{c|}{ PA4 } & \multicolumn{1}{c|}{ PA5 } & PA6 \\
\hline PA1 & 1,000 & & & & & \\
\hline PA2 &, 455 & 1,000 & & & & \\
\hline PA3 &, 544 &, 634 & 1,000 & & & \\
\hline PA4 &, 555 &, 727 &, 745 & 1,000 & & \\
\hline PA5 &, 286 &, 329 &, 259 &, 467 & 1,000 & \\
\hline PA6 &, 491 &, 717 &, 443 &, 721 &, 408 & 1,000 \\
\hline
\end{tabular}

Tabla 16 Correlación de ítems Afectos Positivos, componente de Bienestar Subjetivo

Fuente: elaboración propia, (2019)

\begin{tabular}{|c|c|c|c|c|c|c|}
\hline & NE1 & NE2 & NE3 & NE4 & NE5 & NE6 \\
\hline NE1 & 1,000 & & & & & \\
\hline NE2 & 788 & 1,000 & & & & \\
\hline NE3 & 790 &, 785 & 1,000 & & & \\
\hline NE4 & ,598 & 630 & 7742 & 1,000 & & \\
\hline NE5 & 202 & 222 & 236 & 139 & 1,000 & \\
\hline NE6 & 337 & 399 & ,367 & 334 & ,385 & 1,000 \\
\hline
\end{tabular}

Tabla 17 Correlación de ítems Afectos Negativos, componente de Bienestar Subjetivo

Fuente: elaboración propia, (2019)

\begin{tabular}{|c|c|c|c|c|c|c|}
\hline \multicolumn{2}{|c|}{ L1 } & L2 & L3 & L4 & L5 & L6 \\
\hline L1 & 1,000 & & & & & \\
\hline L2 &, 250 & 1,000 & & & & \\
\hline L3 &, 097 &, 181 & 1,000 & & & \\
\hline L4 &,- 251 &, 237 &, 300 & 1,000 & & \\
\hline L5 &,- 138 &, 072 &, 340 &, 728 & 1,000 & \\
\hline L6 &, 139 &, 196 &, 558 &, 546 &, 766 & 1,000 \\
\hline
\end{tabular}

Tabla 18 Correlación de ítems Lierazgo, componente de Percepción de abuso

Fuente: Elaboración propia (2019).

\begin{tabular}{|c|c|c|c|c|c|c|c|}
\hline & AP1 & APx3 & $\mathrm{APx} 4$ & \multicolumn{2}{|c|}{ APx5 } & $\mathrm{APx} 7$ & AP8 \\
\hline AP1 & 1,000 & & & & & & \\
\hline $\mathrm{APx} 3$ & 0,121 & 1,000 & & & & & \\
\hline $\mathrm{APx} 4$ & 0,166 & 0,938 & 1,000 & & & & \\
\hline APx 5 & 0,152 & 0,798 & 0,856 & & 1,000 & & \\
\hline $\mathrm{APx} 7$ & 0,232 & 0,621 & 0,583 & & 0,478 & 1,000 & \\
\hline AP8 & $-0,229$ & $-0,242$ & $-0,227$ & & $-0,239$ & $-0,130$ & 1,000 \\
\hline & AP9 & AP10 & AP11 & AP12 & AP13 & AP14 & AP15 \\
\hline AP9 & 1,000 & & & & & & \\
\hline AP10 & 0,523 & 1,000 & & & & & \\
\hline AP11 & $-0,333$ & $-0,412$ & 1,000 & & & & \\
\hline AP12 & $-0,304$ & $-0,314$ & 0,519 & 1,000 & & & \\
\hline $\mathrm{AP} 13$ & $-0,202$ & $-0,227$ & 0,431 & 0,054 & 1,000 & & \\
\hline AP14 & $-0,449$ & $-0,629$ & 0,311 & 0,156 & 0,357 & 1,000 & \\
\hline AP15 & $-0,263$ & $-0,295$ & 0,258 & 0,156 & 0,461 & 0,385 & 1,000 \\
\hline
\end{tabular}

Tabla 19 Correlación de ítems Percepción de abuso Fuente: elaboración propia (2019)

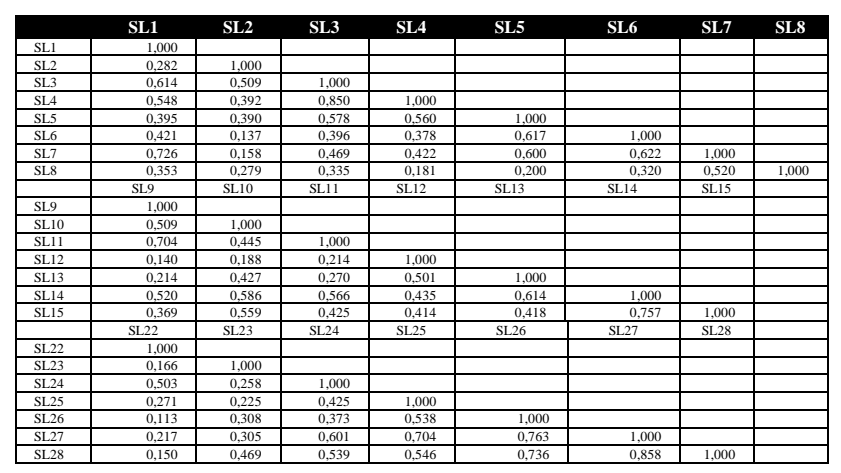

Tabla 20 Correlación de ítems Satisfacción laboral Fuente: elaboración propia, (2019)

Con relación a la confiabilidad del instrumento presentado en escala de Likert, se puede aseverar que no todos los valores obtenidos son consistentes, en algunas secciones, específicamente en el análisis de la Percepción del abuso del poder, la cual presenta valores muy por debajo del 0.7 recomendado por Cronbach, 1951; Nunnaly, 1978; Hair, Anderson, Tatham y Black, 1999.

\begin{tabular}{|r|l|r|}
\hline \multicolumn{3}{|c|}{ Escala de satisfacción con la vida SWLS, Alpha por dimensión. } \\
\hline Alfa de Cronbach & $\begin{array}{l}\text { Alfa de Cronbach } \\
\text { basada en elementos } \\
\text { estandarizados }\end{array}$ & N de elementos \\
\hline 0,698 & 0,752 & 5 \\
\hline
\end{tabular}

Tabla 21 Alpha Satisfacción con la vida Fuente: elaboración propia, (2019)

\begin{tabular}{|r|l|l|}
\hline \multicolumn{3}{|c|}{ Escala de afectos positivos, Alpha por dimensión. } \\
\hline Alfa de Cronbach & $\begin{array}{l}\text { Alfa de Cronbach } \\
\text { basada en elementos } \\
\text { estandarizados }\end{array}$ & N de elementos \\
\hline 0,859 & 0,866 & \\
\hline
\end{tabular}

Tabla 22 Alpha PA

Fuente: elaboración propia, (2019)

\begin{tabular}{|r|r|r|}
\hline \multicolumn{2}{|c|}{ Escala de afectos negativos, Alpha por dimensión. } \\
\hline & $\begin{array}{c}\text { Alfa de Cronbach } \\
\text { basada en elementos } \\
\text { estandarizados }\end{array}$ & N de elementos \\
\hline 0,823 & 0,838 & \\
\hline
\end{tabular}

Tabla 23 Alpha NE

Fuente: elaboración propia, (2019) 


\begin{tabular}{|r|l|l|}
\hline \multicolumn{3}{|c|}{ Escala de satisfacción laboral, Alpha por dimensión. } \\
\hline Alfa de Cronbach & $\begin{array}{l}\text { Alfa de Cronbach } \\
\text { basada en elementos } \\
\text { estandarizados }\end{array}$ & N de elementos \\
\hline 0,931 & 0,934 & 28 \\
\hline
\end{tabular}

Tabla 24 Alpha SL

Fuente: elaboración propia, (2019)

\begin{tabular}{|r|l|l|}
\hline \multicolumn{3}{|c|}{ Escala de percepción de liderazgo, Alpha por dimensión. } \\
\hline Alfa de Cronbach & $\begin{array}{l}\text { Alfa de Cronbach } \\
\text { basada en elementos } \\
\text { estandarizados }\end{array}$ & N de elementos \\
\hline 0,695 & \multicolumn{2}{|c|}{0,687} \\
\hline
\end{tabular}

Tabla 25 Alpha L

Fuente: elaboración propia, (2019)

\begin{tabular}{|r|l|l|}
\hline \multicolumn{2}{|c|}{ Escala de percepción de abuso del poder, Alpha por dimensión. } \\
\hline Alfa de Cronbach & $\begin{array}{l}\text { Alfa de Cronbach } \\
\text { basada en elementos } \\
\text { estandarizados }\end{array}$ & N de elementos \\
\hline 0,252 & 0,517 & 13 \\
\hline
\end{tabular}

Tabla 26 Alpha AP

Fuente: elaboración propia, (2019)

\section{Anexos}

Se muestran los 67 ítems del instrumento aplicado en el presente estudio.

\section{Items del Instrumento}

SWL 1 En la mayoría de los aspectos, mi vida está cerca de mi ideal

SWL 2 Las condiciones de vida son excelentes

SWL 3 Estoy satisfecho con mi vida

SWL 4 Hasta ahora he conseguido las cosas que para mí son importantes en la vida

SWL 5 Si volviera a nacer no cambiaría nada de mi vida En las últimas cuatro semanas he tenido sentimientos...

PA 1 ...positivos

NE $1 \ldots$ negativos

PA 2...buenos

NE 2...malos

PA 3 ... agradables

NE 3 ... desagradables

PA 4...felices

NE 4...tristes

NE 5...de miedo

PA 5...alegres

NE 6...de enfado

PA 6...de satisfacción

SL 1 El nivel jerárquico del cargo que ocupa.

SL 2 El nivel jerárquico del cargo que otros ocupan.

SL 3 Las oportunidades que le ofrece el trabajo actual de desarrollar las actividades que más prefiere.

SL 4 Las oportunidades que le ofrece el trabajo de realizar las actividades en que destaca.

SL 5 La consideración de sus opiniones en las instancias de planificación.

SL 6 La participación en la implementación de lo planificado. SL 7 La participación en instancias superiores (sea con participación directa o indirecta, en órganos de conducción) SL 8 El grado de acuerdo con objetivos y cronograma que guían su desempeño.

SL 9 Las normas vigentes con vinculación directa al desarrollo de su trabajo.

SL $10 \mathrm{La}$ calidad, frecuencia y vías a través de las cuales recibe información relevante para el desarrollo adecuado de su trabajo.

SL 11 Los mecanismos vigentes de control del desempeño de sus funciones.

SL 12 Las condiciones físicas en las que desarrolla su labor.

SL 13 El acceso a recursos pedagógicos.

SL 14 La manera en que se desarrolla el proceso de asignación de cargos.

SL 15 Sus perspectivas de ascenso hacia cargos de mayor jerarquía.

SL 16 La remuneración y prestaciones laborales que recibe como consecuencia de su trabajo, en función a las tareas que realiza y de su formación.

SL 17 Los alicientes recibidos por parte de la Institución que lo hacen sentir apreciado.

SL 18 La capacitación que ha recibido en el pasado.

SL 19 La capacitación que recibe actualmente.

SL 20 El desempeño intrínseco del rol de docente (tareas que realiza independientemente de su percepción respecto a la Cátedra o proyecto particular en el que participa)

SL 21 La posibilidad de ejercer su profesión de forma independiente $o$ en otras organizaciones aparte de su responsabilidad con la Institución.

SL 22 La disposición de tiempo para actividades de esparcimiento fuera de su horario de trabajo.

SL 23 El respeto existente por la diversidad ideológica, racial, sexual, etc. en la Institución.

SL 24 El compartir tiempo libre con los compañeros fuera de la Institución.

SL 25 Las actividades laborales realizadas.

SL 26 Me gusta mi trabajo más que los trabajos que realizan otras personas.

SL 27 El entusiasmo que genera el trabajo.

SL 28 La satisfacción que genera el trabajo.

L1 Valoran el esfuerzo de sus colaboradores

L2 Buscan mantener su imagen

L3 Son Inaccesibles

L4 Tienen favoritismos

L5 Son Autoritarios

L6 Son Descorteses

AP $1 \mathrm{Su}$ jefe le indica realizar actividades que no corresponden al puesto desempeñado

AP 2 Su jefe le indica realizar actividades ilegales y en contra de su voluntad

AP 3 Ha sido avergonzado en público

AP 4 Ha sido avergonzado en privado

AP 5 Ha sido objeto de hostigamiento verbal

AP 6 Ha correspondido involuntariamente a hostigamiento sexual

AP $7 \mathrm{Su}$ jefe realiza actividades intencionales para que obtenga un bajo desempeño

AP 8 Usted obtiene el reconocimiento conforme al desempeño

AP 9 Usted obtiene las oportunidades de promoción por el desempeño

AP 10 Su esfuerzo es valorado

AP 11 Asignación de recursos sin criterios claros

AP 12 Promociones de salario o ascenso no claros

AP 13 Presión para realizar trabajos

AP 14 Temor a la estabilidad en el empleo

AP 15 Ambiente de autoritarismo

Tabla 27 Instrumento de medición de BS, SL, y AP.

Fuente: elaboración propia, (2019) 


\section{Agradecimientos}

Se agradece al personal docente universitario de tiempo completo y a la Institución educativa por las facilidades para realizar el pilotaje.

\section{Conclusiones y recomendaciones}

Como limitantes del estudio se tienen a: la muestra, obtenida por conveniencia y en una sola Institución, por lo que no es posible generalizar resultados, la reducción de ésta debido a la limpieza de datos por los múltiples atípicos que se presentaron, principalmente en Abuso del poder. Tras la revisión teórica del estado del arte, es posible concluir que se requiere continuar con la lectura y fortalecer la base metodológica de las tres variables, incluso ir migrando a conceptos de mayor actualidad en el área de percepción de abuso del poder para hacer posible efectuar distintas pruebas como requisito para aplicar el Análisis Factorial Exploratorio (AFE) y el Análisis Factorial Confirmatorio (AFC), y aplicar el Modelo de Ecuaciones Estructurales (MEE).

\section{Referencias}

Alan B. Krueger and David A. Schkadehe (2007). The Reliability of Subjective WellBeing Measures NBER Working Paper No. 13027 JEL No. C0,I20,J24,J28

Albuquerque, I., de Lima, M. P., Figueiredo, C., \& Matos, M. (2012). Subjective well-being structure: Confirmatory factor analysis in a teachers' Portuguese sample. Social indicators research, 105(3), 569-580.

Albuquerque, I., de Lima, M. P., Matos, M., \& Figueiredo, C. (2014). Work matters: Work personal projects and the idiosyncratic linkages between traits, eudaimonic and hedonic wellbeing. Social indicators research, 115(3), 885906.

Beytía, Pablo y Calvo, Esteban (2011). ¿Cómo Medir La Felicidad? (How to Measure Happiness?) Claves de Políticas Públicas \#4, Public Policy Institute at Universidad Diego Portales, Santiago, Chile. Available at SSRN: https://ssrn.com/abstract=2302809 or http://dx.doi.org/10.2139/ssrn.2302809
Blanch, J. M., Sahagún, M., Cantera, L., \& Cervantes, G. (2010). Cuestionario de bienestar laboral general: estructura y propiedades psicométricas. Revista de Psicología del Trabajo y de las Organizaciones, 26(2), 157-170.

Blanch, Josep M., Sahagún, Miguel, Cantera, Leonor, \& Cervantes, Genís. (2010). Cuestionario de Bienestar Laboral General: Estructura y Propiedades Psicométricas. Revista de Psicología del Trabajo y de las Organizaciones, 26(2), 157-170. Recuperado en 05 de febrero de 2019, de http://scielo.isciii.es/scielo.php?script=sci_artte xt\&pid=S1576$59622010000200007 \& \operatorname{lng}=\mathrm{es} \& \operatorname{tlng}=\mathrm{es}$.

Campos, E. M. M., González, A. F., \& Jacott, L. (2018). Bienestar subjetivo y satisfacción vital del profesorado. REICE. Revista Iberoamericana sobre Calidad, Eficacia y Cambio en Educación, 16(1).

Cenkseven-Onder, F., \& Sari, M. (2009). The Quality of School Life and Burnout as Predictors of Subjective Well-Being among Teachers. Educational Sciences: Theory and Practice, 9(3), 1223-1235.

MG, (1975). The concept of happiness, Doctoral dissertation, University of Michigan, 1974.

Cols, L. J. P. (2016). Satisfacción Laboral en docentes universitarios: medición y estudio de variables influyentes. REDU: Revista de Docencia Universitaria, 14(2), 261-292.

Cronbach, L. J. (1951). Coefficient alpha and the internal structure of tests. psychometrika, 16(3), 297-334.

Diener, E., Oishi, S., \& Lucas, R. E. (2003). Personality, culture, and subjective well-being: Emotional and cognitive evaluations of life. Annual review of psychology, 54(1), 403-425.

Drewnowski, J. (1980). Social indicators, quality of life and economic theory a suggestion for establishing a theoretical basis for social indicators and quality of life research. Philosophica, 25(1), 15-32. 
Fernández, M. L. M. (2016). Inteligencia emocional percibida y su relación con la satisfacción y el bienestar subjetivo del profesorado. Inteligencia Emocional y Bienestar II: reflexiones, experiencias profesionales e investigaciones (pp. 660-675). Ediciones Universidad de San Jorge.

Flores Crespo, Pedro; García García, César; (2014). La Reforma Educativa en México. ¿Nuevas reglas para las IES? Revista de la Educación Superior, XLIII (4) OctubreDiciembre, 9-31.

Flores, R., Madero, S., \& Gómez, J. (2009). Abuso de poder jerárquico y consecuencias en el trabajador. Trabajo presentado en X Congreso Anual de la Academia de Ciencias Administrativas AC, San Luis Potosí, México.

Garrido, S., Méndez, I., \& Abellán, J. M. (2013). Analysing the simultaneous relationship between life satisfaction and health-related quality of life. Journal of Happiness Studies, 14(6), 1813-1838.

Guttman, L. (1945). A basis for analyzing testretest reliability. Psychometrika, 10(4), 255-282.

Hamama, L., Ronen, T., Shachar, K., \& Rosenbaum, M. (2013). Links between stress, positive and negative affect, and life satisfaction among teachers in special education schools. Journal of Happiness Studies, 14(3), 731-751.

Horowitz, J. (2016, June). Dimensions of Job Quality, Mechanisms, and Subjective WellBeing in the United States. In Sociological Forum (Vol. 31, No. 2, pp. 419-440).

Hoyt, C. (1941). Test reliability estimated by analysis of variance. Psychometrika, 6(3), 153160.

Ibáñez, B. C. (1994). Pedagogía y Psicología Interconductual. Revista Mexicana de Análisis de la Conducta, 20, 99-112.

Jijena Michel, R. D., \& Jijena Michel, C. E. (2014). El rol moderador de la flexibilidad de horarios sobre la satisfacción docente: aplicación del método de ecuaciones estructurales. Revista Ventana Cientifica, 1, 20.
Jiménez, B. M., Muñoz, A. R., Gamarra, M. M., \& Herrer, M. G. (2007). Assessing workplace bullying: Spanish validation of a reduced version of the Negative Acts Questionnaire. The Spanish Journal of Psychology, 10(2), 449-457.

Kim-Prieto, C., Diener, E., Tamir, M., Scollon, C., \& Diener, M. (2005). Integrating the diverse definitions of happiness: A time-sequential framework of subjective well-being. Journal of happiness Studies, 6(3), 261-300.

Lazo Pérez, M., \& Lamanier Ramos, J (2017). La inteligencia emocional. Mapa, 4(5), 128-132. Recuperado de http://revistamapa.com

Meliá, J.L., \& Peiró, J. M. (1989). El Cuestionario de Satisfacción S10/12: Estructura factorial, fiabilidad y validez [The S10/12 Job Satisfaction Questionnaire: Factorial structure, reliability and validity]. Revista de Psicología del Trabajo y de las Organizaciones, 4 (11), 179187.

Millán, A., Calvanese, N., \& D'Aubeterre, M. (2017). Condiciones de trabajo, estrés laboral, dependencia universitaria y bienestar psicológico en docentes universitarios. REDU. Revista de Docencia Universitaria, 15(1), 195218. doi:https://doi.org/10.4995/redu.2017.6009

Omar, A. (2006). Justicia organizacional, individualismo-colectivismo y estrés laboral. Psicología y salud, 16(2), 207-217.

Perandones González, T. M., \& Herrera Torres, L. (2017). Autoeficacia docente y fortalezas y virtudes personales en docentes de república dominicana. International Journal of Developmental and Educational Psychology, $3(1)$.

Pontin, E., Schwannauer, M., Tai, S., \& Kinderman, P. (2013). A UK validation of a general measure of subjective well-being: the modified BBC subjective well-being scale (BBC-SWB). Health and Quality of Life Outcomes, 11(1), 150.

Silva, A. D., do Céu Taveira, M., Marques, C., \& Gouveia, V. V. (2015). Satisfaction with life scale among adolescents and young adults in Portugal: Extending evidence of construct validity. Social Indicators Research, 120(1), 309-318. 
Straume, L. V., \& Vitters $\varnothing$, J. (2015). Well-being at work: Some differences between life satisfaction and personal growth as predictors of subjective health and sick-leave. Journal of Happiness Studies, 16(1), 149-168

Valverde Berrocoso, J., Fernández Sánchez, M. R., \& Revuelta Domínguez, F. I. (2013). El bienestar subjetivo ante las buenas prácticas educativas con TIC: su influencia en profesorado innovador. 